\title{
Fatores de risco associados à ocorrência de sarna sarcóptica e prevalência em suínos nas fases de crescimento e terminação, na região Sul do Brasil
}

\author{
Risk factors associated with mange ocurrence and prevalence in pigs during the growing and finishing \\ phases, in the South region of Brazil
}

\author{
Doralice Pedroso-de-Paiva ${ }^{1}$ Nelson Morés ${ }^{2}$ Waldomiro Barioni Júnior ${ }^{3}$ \\ Osmar Antônio Dalla Costa ${ }^{4}$ Jurij Sobestianski ${ }^{5}$ Armando Lopes do Amaral ${ }^{6}$
}

\section{RESUMO}

Foi realizada uma pesquisa epidemiológica observacional em 64 rebanhos da região Sul do Brasil, com o objetivo de determinar a prevalência e identificar o conjunto de fatores de risco que melhor explicam a ocorrência de sarna sarcóptica em suínos nas fase de crescimento e terminação. Foi aplicado um protocolo abrangendo variáveis do ecossistema de suínos em criação intensiva e os dados foram analisados por análise de correspondência múltipla identificando-se dez variáveis associadas à ocorrência de sarna: área menor que $0,85 \mathrm{~m}^{2} /$ suíno; volume de ar menor que 3,0mªnimal, manejo da instalação no sistema contínuo; umidade relativa do ar menor de $60 \%$ ou maior de $70 \%$; uso de ração farelada e fornecida à vontade; uso de ração com composição fora das recomendações técnicas; excesso de moscas nas instalações e ocorrência de outras doenças no lote. Estas variáveis são apresentadas em um mapa permitindo visualizar os fatores associados com rebanhos de baixa, média e alta prevalência de sarna. Dos 3.948 animais examinados na linha de abate, 16,64\% apresentaram dermatite papular eritematosa indicativa de sarna sarcóptica.

Palavras-chave: suíno ao abate, Sarcoptes scabiei var suis, sarna sarcóptica, epidemiologia, fatores de risco

\section{ABSTRACT}

An observational epidemiological research was carried out in 64 swine herds in the South region of Brazil. The objective of this study was to determine the prevalence and also to identify the set of risk factors that best explain the occurrence of mange during the growing and finishing phases in swine operations. A protocol was applied enclosing variable of the ecosystem in intensive swine operation. The data were analyzed by multiple correspondence analyses identifying ten variables associated with the occurrence of mange: area less then $0.85 \mathrm{~m}^{2} / \mathrm{pig}$; air volume less then $3.0 \mathrm{~m}^{3} / \mathrm{pig}$, handling of building in the continuous system; air humidity less then $60 \%$ or greater then $70 \%$; use of mashed meal and offered "add libitum" with use of ration with composition is different of the recommendations techniques; excess of flies in building and occurrence of other illness presented in the group of pigs. These variables are shown in a map allowing to visualize the factors associated with group of pigs of low, average and high prevalence of mange. Of the 3.948 pigs examined in the slaughter plant, $16.64 \%$ had presented erythematous papules dermatitis in the skin indicative of mange.

Key words: slaughter swine, Sarcoptes scabiei var suis, mange, epidemiology, risk factors.

\section{INTRODUÇÃO}

A sarna dos suínos causada pelo Sarcoptes scabiei var suis (DE GEER, 1778) tem sido um problema para os produtores de todo mundo. Está presente nas criações a menos que tenham sido tomadas medidas para sua eliminação (DAVIES, 1992), aumentando na medida em que são

${ }^{1}$ Médico Veterinário, PhD, Embrapa Suínos e Aves, CP 21, BR 153, Km 110, Vila Tamanduá, 89700-000, Concórdia-SC. E-mail: doralice@cnpsa.embrapa.br. Autor para correspondência.

${ }^{2}$ Médico Veterinário, MSc, Embrapa Suínos e Aves. E-mail: mores@cnpsa.embrapa.br

${ }^{3}$ Estatístico, MSc, Embrapa Suínos e Aves. E-mail: barioni@cnpsa.embrapa.br

${ }^{4}$ Zootecnista., MSc, Embrapa Suínos e Aves. E-mail: osmar@cnpsa.embrapa.br

${ }^{5}$ Médico Veterinário, DMV, Embrapa Suínos e Aves. E-mail: soby@terra.com.br

${ }^{6}$ Biólogo, MSc, Embrapa Suínos e Aves. E-mail: armando@cnpsa.embrapa.br 
empregados os métodos intensivos de produção (MARTINEAU et al., 1985). Mesmo as propriedades bem manejadas, com excelente esquema sanitário, não estão isentas dos efeitos desse parasito, causando inquietação e extremo desconforto aos animais, reduzindo a amamentação, além de interferir na produção de leite (LEANING, 1988) e depreciar a pele dos animais ao abate.

O custo de medicamentos e de mão de obra podem ser os fatores mais significativos para o controle da sarna (MARTINEAU et al., 1985). No Brasil, GIROTTO et al. (1995) concluíram que a estratégia de controle com aplicação de sarnicida, após diagnóstico por raspado de pele, apresentava menor custo por animal, quando comparada ao custo do uso de produto injetável. $\mathrm{O}$ controle da sarna realizado por SMETS et al. (1998) permitiu uma melhora da performance pelo ganho de $100 \mathrm{~g}$ na conversão alimentar, o que foi traduzido por uma redução de $8 \mathrm{~kg}$ de alimentos para completar o crescimento de animais com média de $80 \mathrm{~kg}(25-105 \mathrm{~kg})$.

O problema está amplamente difundido e suínos de todas as classes, idades e condições são suscetíveis à escabiose (WILLIAMS, 1986). A amostragem em reprodutores de uma estação de quarentena, no Brasil, registrou a ocorrência de sarna em 5,65\% dos animais (LIGNON et al., 1989). No Reino Unido, havia pelo menos um animal positivo para sarna, em $67 \%$ de 70 propriedades de terminação amostradas em 1990 (GARCIA, 1991). Na Holanda, em 400 animais examinados na fase de terminação, foi registrada a prevalência de $8,2 \%$ (HOLLANDERS \& VERCRUYSSE, 1990) e de 27,5\%, no Canadá (HOLLANDERS et al.,1992). A alta prevalência encontrada por LA HORIE (1990) em rebanhos de engorda foi atribuída ao fato das granjas receberem animais de diferentes propriedades com condições sanitárias as mais diversas.

A prevalência de sarna nos rebanhos suínos pode ser estimada através do exame macroscópico da pele de animais abatidos, uma vez que a dermatite papular eritematosa é altamente específica para a sarna sarcóptica (HOLLANDERS et al., 1992). Em matadouro, houve uma associação significativa entre a presença de ácaros na orelha e a contagem de lesões utilizada por MENDEZ de VIGO et al. (1992), porém Mc MULLIN et al. (1990) alertam quanto à possibilidade de ser subestimada a prevalência nesse tipo de levantamento.

Em animais de engorda, uma exposição precoce pode aumentar as perdas pela interferência na performance, mas haverá tempo para a regressão das lesões antes do abate, enquanto uma exposição tardia terá o mínimo efeito sobre a performance, mas resultará em severas lesões ao nível de matadouro (DAVIES et al.,1992).

O presente trabalho teve por objetivo identificar os fatores de risco associados à ocorrência de sarna sarcóptica em suínos nas fases de crescimento e de terminação e avaliar a prevalência em rebanhos dos estados do sul do País.

\section{MATERIAL E MÉTODOS}

O trabalho foi executado no período de outubro de 1995 a fevereiro de 1997. Foram examinados animais provenientes de 19 granjas do Estado de SC; 25 do RS e 20 do PR, totalizando 64 granjas da região sul do Brasil. Em cada granja, foi acompanhado um lote de cerca de 60 animais. Os animais foram examinados individualmente na linha de abate, usando o método descrito por POINTON et al. (1992), com escores de lesão de $0=$ para ausência de lesões; $1=$ lesões brandas localizadas; $2=$ lesões brandas generalizadas e $3=$ lesões severas generalizadas. Em SC, a avaliação foi realizada nos meses de outubro de 95 a junho de 97; no RS de junho de 96 a fevereiro 97 e no PR de junho a dezembro de 97.

Foram avaliadas granjas de Ciclo Completo (CC) e de Terminação nos sistemas de Parceria (TP) e Independentes (TI). As TP e TI recebiam leitões de diversas origens e, nestas, desenvolviam-se as fases de crescimento e terminação dos animais. As granjas de TP eram ligadas a empresas integradoras, recebendo delas a ração já pronta. A ração fornecida pelas integradoras já contém, em sua formulação, produtos anti-helmínticos. De acordo com o tipo de criação as granjas tiveram a seguinte distribuíção por Estado: PR - 9 CC e 11 TP; RS - 14 CC, 7 TP e 4 TI; SC - 9 CC, 6 TP e 4 TI.

Em cada granja, foi aplicado um protocolo com variáveis ligadas aos animais, às instalações, à nutrição, ao manejo e ao ambiente. Foi acompanhada, durante o período de terminação, a população de moscas presente nas instalações (Mosca_I), através de contagem do número de manchas de fezes e/ou de regurgitações deixadas pelas moscas em cartões de $7 \mathrm{x} 14 \mathrm{~cm}$, expostos por 7 dias, além do número de moscas pousadas sobre os animais (Mosca A) em leituras realizadas quinzenalmente, durante 5 minutos, com três repetições.

Para análise dos dados com o objetivo de identificar os fatores de risco, adotou-se a metodologia descrita por MADEC \& JOSSE (1984). As variáveis foram analisadas por métodos descritivos e multidimensionais (Análise Fatorial de 
Correspondência Múltipla - AFCM). Inicialmente definiu-se a variável objetiva (aquela que melhor definia a ocorrência de sarna) e as explicativas (aquelas que se constituíam em potenciais fatores de risco). Essas variáveis foram categorizadas ou seja, foram desmembradas em classes, estabelecendo-se limites de acordo com a experiência do pesquisador, com as informações da literatura ou com a própria distribuição dos dados. O conjunto das variáveis explicativas que apresentaram associação com a ocorrência de sarna $(\mathrm{p} \leq 0,20)$ foi usado na AFCM para a elaboração do mapa de fatores de risco.

A variável resposta foi definida como índice de ocorrência de sarna por granja (SARNA) e foi gerada ponderando os escores de lesão ( 0 a 3 ) e a freqüência de animais por escore (n), utilizando a seguinte fórmula:

$\operatorname{total}(\mathrm{n} 0+\ldots+\mathrm{n} 3)$

SARNA $=(0 * n 0+1 * n 1+2 * n 2+3 * n 3) /$

Os pacotes estatísticos adotados para análise dos dados foram: Statistical Analysis System
(SAS, 1996) e Sistema Portátil de Análise de Dados Numéricos (SPADN) (CISIA, 1995).

\section{RESULTADOS E DISCUSSÃO}

A variável objetiva e as variáveis identificadas como o conjunto de fatores de risco associados à ocorrência de sarna, bem como suas classes e freqüências de rebanhos em cada classe estão descritas na tabela 1 .

$\mathrm{Na}$ definição do mapa pela AFCM foram identificados 10 fatores que melhor explicaram a presença de sarna nos rebanhos acompanhados nas fases de crescimento e terminação (Figura 1).

No quadrante superior direito do mapa, observa-se que estão localizadas classes de variáveis de risco (Mosca_A3, Ur2, Vol3, Doença3 e Lot3) que estão relacionadas com maior índice de ocorrência de sarna (Sarna3). No lado oposto do mapa, no quadrante superior esquerdo, encontram-se as classes de variáveis boas (Vol1, Lot1, Doenca1, Vazio1, Mosca-

Tabela 1 - Variável objetiva e variáveis explicativas associadas à ocorrência de sarna em suínos nas fases de crescimento e terminação.

\begin{tabular}{|c|c|c|c|}
\hline \multirow{2}{*}{ Variável objetiva } & \multirow{2}{*}{ Classes/limite } & \multicolumn{2}{|c|}{ Freqüência de rebanhos } \\
\hline & & $\mathrm{N}$ & $\%$ \\
\hline \multirow{3}{*}{ Ocorrência de sarna (índice) } & SARNA1 $\leq 0,06$ & 19 & 29,7 \\
\hline & SARNA2 $>0,06 \leq 0,20$ & 26 & 40,6 \\
\hline & SARNA3 $>0,20$ & 19 & 29,7 \\
\hline \multicolumn{4}{|c|}{ Variáveis explicativas (fatores de risco) } \\
\hline \multirow{3}{*}{ Densidade (suínos $/ \mathrm{m}^{2}$ ) } & LOT1 $>1,00$ & 22 & 34,4 \\
\hline & LOT $2 \leq 0,85 \leq 1,00$ & 22 & 34,4 \\
\hline & LOT3 $<0,85$ & 20 & 31,2 \\
\hline \multirow{3}{*}{ Volume de ar por suíno $\left(\mathrm{m}^{3}\right)$} & VOL $1>4,50$ & 22 & 34,4 \\
\hline & VOL $2 \leq 3,00 \leq 4,50$ & 23 & 35,9 \\
\hline & VOL3 $<3,00$ & 19 & 29,7 \\
\hline \multirow{2}{*}{ Manejo das instalações } & VAZIO1 = todos dentro todos fora & 26 & 40,6 \\
\hline & VAZIO2 = sistema contínuo & 38 & 59,4 \\
\hline \multirow{2}{*}{ Umidade relativa do ar (9 horas) } & Ur1 entre 60 a 70 & 30 & 46,9 \\
\hline & Ur2 fora destes valores & 34 & 53,1 \\
\hline \multirow{2}{*}{ Forma de apresentação da ração } & FRACAO1 = peletizada & 33 & 51,6 \\
\hline & FRACAO2 = farelada & 31 & 48,4 \\
\hline \multirow{2}{*}{ Manejo da ração na terminação } & MRACAO1 = restrição alimentar & 31 & 48,4 \\
\hline & MRACAO2 = à vontade & 33 & 51,6 \\
\hline \multirow{2}{*}{ Qualidade da ração } & RACAO_B $=$ boa & 35 & 54,7 \\
\hline & RACAO_R $=$ ruim & 29 & 45,3 \\
\hline & MOSCA_A1 $<20$ & 15 & 23,4 \\
\hline \multirow[t]{2}{*}{ Número de moscas nos animais } & MOSCA_A $2 \leq 20<80$ & 22 & 34,4 \\
\hline & MOSCA_A3 $\geq 80$ & 27 & 42,2 \\
\hline \multirow{2}{*}{ Contagem de mosca pelo cartão } & MOSCA_I1 = baixo & 52 & 81,2 \\
\hline & $\operatorname{MOSCA} I 2=$ alto & 12 & 18,8 \\
\hline \multirow{3}{*}{ Presença de doenças } & DOENCA1 = baixa ocorrência & 20 & 31,3 \\
\hline & DOENCA2 = média ocorrência & 26 & 40,6 \\
\hline & DOENCA3 = alta ocorrência & 18 & 28,1 \\
\hline
\end{tabular}

As classes em negrito são consideradas fatores de risco para a sarna. 


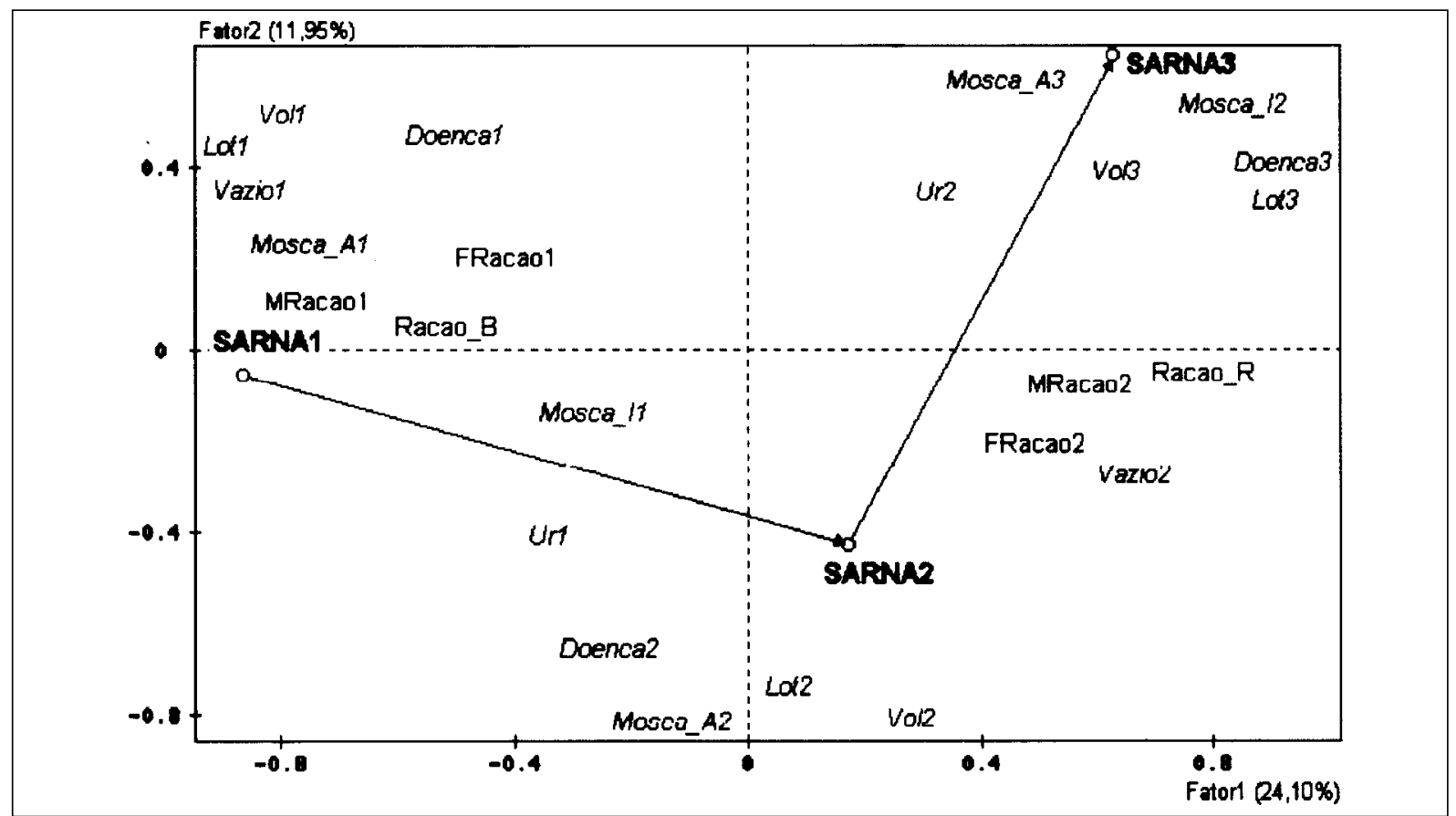

Figura 1 - Mapa dos fatores de risco associados à ocorrência de sarna em suínos nas fases de crescimento e terminação.

A1, FRacao1, MRacao1, Racao_B e Mosca_I1) que estão associadas a um baixo índice de ocorrência de sarna (Sarna1). Nos quadrantes inferiores, mais ao centro do mapa, estão as classes de variáveis mais associadas ao índice intermediário de ocorrência de sarna (Sarna 2).

Dos fatores de risco analisados, a presença de grande número de moscas nas instalações (Mosca_I2) e nos animais (Mosca_A3), aliados aos fatores que caracterizam criações de suínos pouco tecnificadas, como é o caso da não adoção de vazio sanitário (Vazio2), uso de ração farelada (Fracao2), administração de ração à vontade (Mracao2), baixa qualidade na ração por baixos níveis de nutrientes (Racao_R), estiveram aliados à alta ocorrência sarna (SARNA3). Esses fatores também caracterizam grande parte das criações independentes, ou seja, não ligadas a empresas integradoras. Da mesma forma, a alta lotação (Lot3) e volume de ar por suíno abaixo de $3 \mathrm{~m}^{3}$ (Vol3), fatores que podem estar presentes em qualquer sistema de criação, além da alta umidade relativa no ar(Ur2), também apresentaram associação com alta prevalência de sarna (SARNA3). A alta ocorrência de doenças respiratórias (Doenca3), como pneumonia e rinite atrófica, também esteve associada à ocorrência de sarna (SARNA3). Neste caso, não pode ser considerado como um fato de causa e efeito, mas sim de granjas com animais com baixa resistência estando susceptíveis a apresentar sinais clínicos compatíveis com sarna sarcóptica.

Quando essas variáveis estão presentes nos rebanhos de suínos, aumentam as chances de ocorrência de sarna e não devem ser consideradas isoladamente, uma vez que existem inter-relações entre elas.

No estudo da prevalência da doença, observou-se que dos 3.948 animais examinados, $16,64 \%$ apresentavam dermatite papular eritematosa indicativa de sarna (Tabela 2). Essa prevalência é superior à observada por HOLLANDERS \& CASTRYK (1988) na Holanda $(8,2 \%)$, porém está abaixo do

Tabela 2 - Freqüência absoluta de escores de lesão de dermatite relacionada à sarna em animais, provenientes de 64 granjas, abtidos NA região Sul do Brasil e freqüência relativa NA região.

\begin{tabular}{lccccc}
\hline Estado & \multicolumn{3}{c}{ Escores de lesão } & Total de animais \\
& 0 & 1 & 2 & 3 & examinados \\
\hline Paraná & 1197 & 84 & - & - & 1281 \\
Santa Catarina & 869 & 265 & 27 & 1 & 1162 \\
Rio Grande do Sul & 1225 & 243 & 35 & 2 & 1505 \\
TOTAL & 3291 & 592 & 62 & 3 & 3948 \\
Região (\%) & 83,36 & 14,99 & 1,57 & 0,08 & 100 \\
\hline
\end{tabular}

Ciência Rural, v. 33, n. 4, jul-ago, 2003. 
observado por HOLLANDERS et al. (1992), em animais de terminação, no Canadá (27,5\%). Assemelha-se, no entanto, ao observado por KLOPFENSTEIN et al. (1992) em plantel "high-health", também no Canadá $(18,2 \%)$, porém é superior à encontrada em levantamento anterior realizado no Brasil, por LIGNON et al. (1989) de 5,61\%.

Das 64 granjas analisadas, $51(82,3 \%)$ apresentavam pelo menos um animal com dermatite, prevalência esta superior àquela observada no Reino Unido (GARCIA, 1991) indicando alta prevalência nas criações da região sul do Brasil.

As granjas de Terminadores Independentes (TI) nos três Estados, apresentaram maiores percentuais de animais positivos para sarna (Tabela 3 ). Nesse modelo de criação, a produção da ração, em geral, é feita na propriedade e a inclusão de ectoparasiticida fica a critério do produtor, enquanto nas TP, a ração é fornecida pela empresa integradora, geralmente com programas estratégicos de controle de sarna. Deve-se considerar, ainda, o nível de tecnologia adotado nessas granjas, pois o estudo dos fatores de risco demonstrou que a ausência de sarna está associada à alta tecnificação das granjas.
No entanto, quando se verifica a freqüência de lesões de escores 2 e 3 (Tabela 4) que, conforme HOLLANDERS et al. (1992) são "altamente específicas para sarna sarcóptica", observa-se que maior número de animais de granjas de ciclo completo apresentaram tais escores.

Outro fator a se levar em consideração é que, tanto as granjas TI como as $\mathrm{CC}$, ao contrário das TP, usavam o sistema de produção contínuo, sem vazio sanitário e sem formação de lotes por sala, o que favorece a manutenção e disseminação de doenças, inclusive a sarna.

No Paraná, não foram acompanhadas granjas de terminadores independentes, mas em SC e no RS, esse sistema de criação também apresentou escore 2 .

Nos sistemas de criação com maior prevalência, faz-se necessária a tomada de medidas preventivas, com a correção dos fatores de risco, na entrada das estações mais propícias a essa doença, sem deixar de considerar o aspecto econômico da questão (GIROTTO et al., 1995), tanto de higiene das instalações quanto utilização de medicamentos.

Tabela 3 - Freqüência absoluta e relativa de lesão de dermatite relacionada à sarna em animais abatidos nas região Sul do Brasil, de acordo com o tipo de criação.

\begin{tabular}{|c|c|c|c|c|c|c|c|c|c|}
\hline \multirow{2}{*}{ ESTADO } & \multicolumn{3}{|c|}{ Ciclo Completo } & \multicolumn{3}{|c|}{ Terminação Independente } & \multicolumn{3}{|c|}{ Terminação Parceria } \\
\hline & $\mathrm{n}$ & + & $\%$ & $\mathrm{n}$ & + & $\%$ & $\mathrm{n}$ & + & $\%$ \\
\hline Paraná & 540 & 57 & 10,26 & - & - & - & 741 & 27 & 3,64 \\
\hline Santa Catarina & 565 & 142 & 25,13 & 235 & 57 & 24,26 & 362 & 94 & 25,96 \\
\hline Rio Grande do Sul & 857 & 161 & 18,78 & 218 & 59 & 27,07 & 430 & 60 & 13,95 \\
\hline TOTAL & 1962 & 360 & 18,34 & 453 & 116 & 25,61 & 1533 & 181 & 11,81 \\
\hline
\end{tabular}

$\mathrm{n}=$ Número de animais abatidos; $+=$ Número de animais positivos para dermatite papular relacionada à sarna;

$\%=$ Percentual de animais positivos para dermatite papular.

Tabela 4 - Freqüência absoluta e relativa de escores de lesão de dermatite relacionada à sarna em animais abatidos nas região Sul do Brasil, provenientes de 64 granjas.

\begin{tabular}{|c|c|c|c|c|c|c|c|c|c|c|c|c|}
\hline \multirow{3}{*}{ ESTADO } & \multicolumn{4}{|c|}{ Ciclo Completo } & \multicolumn{4}{|c|}{ Terminação independente } & \multicolumn{4}{|c|}{ Terminação parceria } \\
\hline & \multicolumn{4}{|c|}{ Escores de lesão* } & \multicolumn{4}{|c|}{ Escores de lesão } & \multicolumn{4}{|c|}{ Escores de lesão } \\
\hline & 0 & 1 & 2 & 3 & 0 & 1 & 2 & 3 & 0 & 1 & 2 & 3 \\
\hline Paraná & 483 & 57 & 0 & 0 & - & - & - & - & 714 & 27 & 0 & 0 \\
\hline Santa Catarina & 423 & 126 & 15 & 1 & 178 & 52 & 5 & 0 & 268 & 87 & 7 & 0 \\
\hline Rio Grande do Sul & 696 & 133 & 26 & 2 & 159 & 50 & 9 & 0 & 370 & 60 & 0 & 0 \\
\hline TOTAL & 1602 & 316 & 41 & 3 & 337 & 102 & 14 & 0 & 1352 & 174 & 7 & 0 \\
\hline Percentual & 81,6 & 16,1 & 2,1 & 0,2 & 74,4 & 22,5 & 3,1 & 0,0 & 88,2 & 11,4 & 0,5 & 0,0 \\
\hline
\end{tabular}

* Escores de lesão: 0 = ausência; 1 = lesões brandas localizadas; 2 = lesões brandas generalizadas;

$3=$ lesões severas generalizadas. 


\section{CONCLUSÃO}

Conclui-se que a terminação de suínos em sistema de produção contínuo, sem vazio sanitário, com alta lotação, volume e umidade relativa do ar inadequados, com fornecimento de ração de má qualidade, farelada e à vontade, além da presença de grande população de moscas e alta ocorrência de doenças são fatores de risco associados à ocorrência de sarna.

\section{AGRADECIMENTOS}

Aos 64 produtores de suínos, por colocarem suas granjas à disposição para o estudo e pela colaboração nas anotações de dados. Aos técnicos das agroindústrias e da extensão rural, que colaboraram na identificação dos rebanhos e na aplicação dos protocolos.

\section{REFERÊNCIAS BIBLIOGRÁFICAS}

CENTRE INTERNACIONAL DE STATISTIQUE ET D'INFORMATIQUE APPLIQUÉES - CISIA (Saint-Mandé, France) Oferta estudante SPADN integrado: version P.C. Saint-Mandé, 1995. 215p.

DAVIES, P.R. et al. Are skin lesions at slaughters useful for evaluatin sarcoptic mange in pigs? In: INTERNATIONAL PIG VETERINARY SOCIETY, 12., 1992, The Hague. Proceedings... The Hague : IPVS, 1992. p 371.

DAVIES, P.R. Assessing the severity of sarcoptic mange in growing swine. In: INTERNATIONAL PIG VETERINARY SOCIETY, 12., 1992, The Hague. Proceedings... The Hague : IPVS, 1992. p.370.

GARCIA, R. Health special: losses from skin mites. Pig International, v.21, n.11, p.12 e 14, 1991.

GIROTTO, A.F. et al. Sarna sarcóptica dos suínos. II. Avaliação econômica de estratégias alternativas no controle. Pesquisa Agropecuária Brasileira, v.30, n.1, p.125-129, 1995.

HOLLANDERS, N.; CASTRYCK, F. Survey of the prevalence of Sarcoptes sacabiei in fatteners in Belgium. In: INTERNATIONAL PIG VETERINARY SOCIETY, 10., 1988, Rio de Janeiro. Proceedings... Rio de Janeiro : IPVS, 1988. p.255.

HOLLANDERS, N; VERCRUYSSE, J. Sarcoptic mite hypersensitivity: a cause of der-matitis in fattening pigs at slaughter. The Veterinary Record, v.126, p.308-310, 1990.

HOLLANDERS, N.; HENRIKSEN, S.A.; EBBESEN, T.J.
Sarcoptes scabiei infestation and generalized papuladermatitis in Danish slaughter pigs. In: INTERNATIONAL PIG VETERINARY SOCIETY, 12., 1992, The Hague. Proceedings... The Hague : IPVS, 1992. p.372.

KLOPFENSTEIN, C. et al. Survey of the prevalence of sarcoptica mange and dermatitis in slaughter pigs in Quebec, Canada. In: INTERNATIONAL PIG VETERINARY SOCIETY, 12., 1992, The Hague. Proceedings... The Hague : IPVS, 1992. p.376.

LA HORIE, P.F. de. Prevalence of sarcoptic mange in fattening pigs results of a french survey. In: INTERNATIONAL PIG VETERINARY SOCIETY, 11., 1990, Lauzanne. Proceedings... Lauzanne : IPVS, 1990. p.320.

LEANING, W.H.D. Studies of the benefits of administering ivermectin for parasite control in sows before farrowing. In: INTERNATIONAL PIG VETERINARY SOCIETY, 10., 1988, Rio de Janeiro. Proceedings... Rio de Janeiro : IPVS, 1988. p.264.

LIGNON, G.B. et al. Sarna sarcóptica dos suínos: estratégia de ação no controle. In: CONGRESSO ABRAVES, 4., 1989, Itapema, Anais... Itapema : ABRAVES, 1989. p.101.

MADEC, F.; JOSSE, J. Utilization des methodes d'analyse des donnes pour l'etude de maladies d'alevage application du porc. Epidemiologie Sante Animall, v.6, p.35-63,1984.

MARTINEAU, G.P.; VAILLANCOURT, J.; FRÉCHETTE, J.L. O controle da infestaçäo por Sarcoptes scabiei com Ivermectin, numa grande criação intensiva suína de reprodução. Revista Brasileira de Medicina Veterinária, v.7, n.6, p.171-174, 1985.

Mc MULLIN, P.F. et al. A survey of sarcoptic mange in cull sows in Great Britain. In: INTERNATIONAL PIG VETERINARY SOCIETY, 11., 1990, Lauzanne. Proceedings... Lauzanne : IPVS, 1990. p.318.

MÉNDEZ DE VIGO, J. et al. Study of the prevalence of sarcoptic mange in pigs at slaughterhouses in major swineproduction areas in Spain. I n: INTERNATIONAL PIG VETERINARY SOCIETY, 12., 1992, The Hague. Proceedings... The Hague : IPVS, 1992. p. 374.

POINTON, A.M. et al. Disease surveillance at slaughter. In: LEMAN, A.D.et al. Diseases of swine. 7. ed. Ames, Iowa, USA : Iowa State University, 1992. 1021p.

SAS INSTITUTE INC. System for Microsoft Windows, release 6. 12. Cary, NC, USA, 1996. 1 CD ROM.

SMETS, K.; NEIRYNCK, W.; VERCRUYSSE, J. Mange eradication. Part. 1. An important aspect of quality assurance... Belgian eradication objective. P ig Progress, v.14, n.8, p.2830, 1998.

WILLIAMS, R.E. Epidemiology and control of ectoparasites of swine. Veterinary Clinics of North America: Food Animal Practice, v.2, n.2, p.469-479, 1986. 